\title{
DESARROLLO HUMANO Y CRECIMIENTO ECONÓMICO EN EL ÁMBITO REGIONAL COLOMBIANO (1990-2005)
}

\author{
Carlos Andrés Gualdrón Guerrero*
}

\footnotetext{
* Docente Investigador tiempo completo Facultad de Ciencias Económicas y Empresariales Universidad de Pamplona. Pamplona Colombia. E-mail: carlosgualdron@unipamplona.edu.co
} 


\section{DESARROLLO HUMANO Y CRECIMIENTO ECONÓMICO EN EL ÁMBITO REGIONAL COLOMBIANO (1990-2005)}

\section{RESUMEN}

En la presente investigación se analizan las relaciones entre desarrollo humano y crecimiento económico en el ámbito regional colombiano. Para ello, adaptamos los métodos de estudio propuestos durante los últimos años por Ranis, Stewart y Ramírez en los que consideran la existencia de cuatro situaciones distintas en la relación entre ambas variables: círculos viciosos, círculos virtuosos, sesgos procrecimiento y sesgos pro-desarrollo. Aplicamos el método a escala regional para todas las regiones colombianas.

Palabras Claves: Desarrollo humano, crecimiento económico, círculo vicioso, círculo virtuoso, sesgos pro-crecimiento y sesgos prodesarrollo.

\begin{abstract}
In the present investigation the relations are analyzed between human development and economic growth in the regional Colombian area. For it, we adapt the methods of study proposed during the last years by Ranis, Stewart and Ramirez in those who consider the existence of four different situations in the relation between both variables: vicious circles, virtuous circles, biases pro-growth and biases I pro-develop. We apply the method to regional scale for all the Colombian regions.
\end{abstract}

Key Words: human develop, economic development, vicious circles, virtuous circles, biases pro-growth and biases I pro-develop.

\section{Face IssN 1794-9920}

Recepción: Abril de 2008

Revisión: Mayo de 2008

Aceptación: Mayo de 2008 


\section{INTRODUCCIÓN}

En las últimas décadas se ha debatido la relación existente entre crecimiento económico y desarrollo, de tal manera que aún siendo frecuente la conclusión de que puede existir crecimiento sin desarrollo parece también generalizada la idea en la que se considera necesario el crecimiento económico para lograr el desarrollo.

El crecimiento económico genera desarrollo humano a medida que la base de recursos se amplíe y exista una buena distribución de los mismos, que permita mejorar interrumpidamente el bienestar de la sociedad, o a medida que estos recursos provenientes del ingreso nacional se asignen a actividades que contribuyan a el desarrollo; y el desarrollo humano genera más crecimiento a medida que una población más sana y educada contribuye a mejorar el desempeño económico (Ranis, Stewart y Ramírez, 2002).

Al respecto, diversos estudios empíricos en los últimos años han puesto en tela de juicio la supuesta relación automática entre crecimiento económico y desarrollo humano, y han corroborado la necesidad de dar prioridad al desarrollo humano para alcanzar un ciclo virtuoso. Así se constata en Ranis, Stewart y Ramírez, 2002 y PNUD, 1996. Precisamente el objetivo principal de este trabajo es incorporar más evidencia empírica sobre la cuestion, y trataremos de ver si esas mismas conclusiones siguen vigentes en Colombia y su ámbito regional para el periodo $1990-2005$.

\section{RELACIÓN CRECIMIENTO Y DESARROLLO HUMANO}

\section{Antecedentes}

El PIB es uno de los índices que sirven para elaborar el $\mathrm{IDH}^{1}$, lo que apunta a una correlación muy estrecha entre el $\mathrm{PIB} / \mathrm{H}$ y el IDH, pero la evidencia empírica muestra que existen países donde esa correlación está sesgada y presenta un desequilibrio entre los valores esperados de las variables.

Así, Ranis, Stewart y Ramírez (2000) utilizaron regresiones comparadas que abarcaron entre 35 y 76 países en desarrollo, según los datos disponibles sobre educación y salud para el periodo 1960-1992, y encontraron:

a).El crecimiento del PIB per cápita resultó ser significativo y bastante sólido en todas las ecuaciones, y el mayor crecimiento del mismo se tradujo en un mejor comportamiento del desarrollo humano.

${ }^{1}$ El IDH es el índice utilizado por el PNUD como medida del desarrollo humano por ser el índice más disponible y que reúne en mayor medida las variables de interés de este trabajo. Sin embargo, el desarrollo humano contiene más aspectos como la igualdad de géneros, entre otros. 
b) La proporción de recursos nacionales destinados a gasto social casi siempre resultó ser significativamente positiva.

c) El comportamiento del hogar derivado de los ingresos de las mujeres, sus conocimientos y el control que ejercían dentro del hogar, aumentó la tasa de matricula femenina en la enseñanza primaria.

d) Contrariamente a lo previsto, la distribución más equitativa del ingreso no pareció influir en el IDH.

Considerando como variable dependiente el PIB per cápita en el periodo 1970-1992 el trabajo de Ranis, Stewart y Ramírez dio los siguientes resultados para los países en vía de desarrollo:

a) Las medidas del grado inicial de desarrollo humano eran invariablemente significativas para explicar el crecimiento del PIB, aunque con coeficientes bajos.

b) Con una sola excepción, la variación de la esperanza de vida (1962-1982) era también positiva y significativa

c) La distribución más equitativa del ingreso iba unida a un mayor crecimiento económico.

Para clasificar empíricamente el comportamiento de los países, Ranis, Stewart y Ramírez compararon los resultados de cada uno con el promedio de todos los países en desarrollo, denominando las posiciones extremas como sesgo prodesarrollo económico (mayor incremento del IDH que la media y menor variación del PIB/H que la media) y como sesgo procrecimiento económico (mayor variación en $\mathrm{PIB} / \mathrm{H}$ que la media ante un rendimiento del IDH menor que la media).

La conclusión más interesante es que ninguno de los países que comenzó con un sesgo procrecimiento económico pudo pasar de allí a un ciclo virtuoso, esto es de mayores crecimientos del PIB/habitante y del IDH que la media del conjunto de países analizados, y todos ellos volvieron a caer en el círculo vicioso (esto es de menores crecimientos de ambas variables que la media del conjunto de países analizados). En cambio, algunos países que comenzaron con un sesgo pro desarrollo humano, efectivamente lograron avanzar hasta situarse en el grupo de círculo virtuoso.

Otro de los resultados de estos autores, es que existen conexiones en Latinoamérica mas débiles que a nivel mundial cuando van del crecimiento económico al desarrollo humano y mas fuertes cuando van del desarrollo humano al crecimiento económico.

Distintas investigaciones han ampliado y corroborado los resultados generales obtenidos en el trabajo de Ranis, Stewart y Ramírez, destacando los efectos positivos sobre el crecimiento económico que tienen la mejora del nivel educativo y de la salud en los países en vía de desarrollo. Así, entre otros: 
a) La ampliación de la educación primaria aumenta la productividad de los trabajadores urbanos y rurales. Además, la educación contribuye de manera importante a la capacidad técnica y al cambio tecnológico en la industria. ${ }^{2}$

b) Se ha demostrado que el mejoramiento de la salud y de la nutrición influye directamente en la productividad de la mano de obra, especialmente en las personas más pobres. Incluso en algunos casos, la evidencia indica que la salud y la nutrición influyen aun más en la productividad que la educación formal. ${ }^{3}$

c) La enseñanza secundaria, incluso la técnico-profesional, facilita la adquisición de destrezas y de capacidad de gestión.

d) La enseñanza terciaria contribuye al desarrollo de la ciencia básica, a la selección adecuada de las importaciones de tecnología, a su adaptación al medio nacional y al desarrollo de tecnologías autóctonas.

e) La enseñanza secundaria y terciaria son elementos fundamentales para el desarrollo de instituciones claves, las cuales son esenciales para el desarrollo económico.

f) Varios estudios empíricos han mostrado que la educación tiene efectos positivos en el crecimiento a nivel macroeconómico, los que varían según el grado de introducción y el modelo de crecimiento macroeconómico elegido. ${ }^{4}$

g) La educación afecta la naturaleza y el crecimiento de las exportaciones que, a su vez, influyen en la tasa global de crecimiento. ${ }^{5}$

h) La educación también puede influir en el incremento del ingreso per cápita a través de sus efectos en el denominador. Muchos estudios han demostrado que la escolaridad femenina está inversamente relacionada con la fecundidad (Ainsworth, Beegle y Nyamete, 1995).

i) También se ha demostrado que ampliar la enseñanza primaria conduce a una distribución más equitativa del ingreso (Psacharopoulos y otros 1992, p.48) y estudios recientes indican que la distribución más equitativa de bienes e ingresos contribuye al crecimiento económico. ${ }^{6}$

En el sentido de la influencia del desarrollo humano sobre el crecimiento económico, (Lapito, 2004) argumenta que se trata de promover la potenciación de la capacidad de la gente no sólo como factor de producción, con aumento de los conocimientos, la productividad y los salarios, sino además, como un fin en si mismo, procurando la equidad en la oferta de las oportunidades. Desde esta perspectiva, se plantea, como punto neurálgico, la atención de los grupos de la población en condiciones de vulnerabilidad socio-económica, los cuales requieren de más ayuda estatal que los

\footnotetext{
2 Véase Shultz (1975), Welch (1970), Rosenzweing (1995), Foster y Rosenzweing (1994).

3 Véase las encuestas de Behrman, 1993 y 1996.

${ }^{4}$ Las nuevas teorías sobre el crecimiento aspiran a hacer endógeno el proceso técnico con la incorporación de algunos de estos mismos efectos, haciendo hincapié a la vez en la educación en el aprendizaje con la práctica y en investigación y desarrollo. Por ejemplo, Barro (1991) y Barro y Lee (1993 a y b).

${ }^{5}$ Entre otros, Michaely (1977), Kruger (1978), Ram (1985), Rama (1998) y Edwards (1993).

6 Véase Asesina y Rodrik (1994), Asesina y Perotti (1994), Persson y Trabellini (1994) y Birdsall, Ross y Sabor (1995).
} 
grupos en situación privilegiada, y el énfasis del gasto en servicios sociales básicos y en programas sociales focalizados.

Autores como (La Fuente y Sáenz, 2001) comentan que el crecimiento económico no necesariamente es bueno para reducir la pobreza, que es uno de los aspectos del desarrollo humano, ya que en sus conclusiones, llama la atención el escaso porcentaje de los ingresos que captan los grupos pobres, debido a la mala distribución de la riqueza en América Latina.

Finalmente en el informe sobre Desarrollo Humano del PNUD de 1996 se analiza el carácter y el grado de fortaleza de los vínculos entre el crecimiento económico y el desarrollo humano. Hay dos conclusiones importantes en este informe. En los 15 años anteriores a este informe, el crecimiento venia fracasando en 100 países, donde vivía casi un tercio de la población del mundo. La otra conclusión es que los vínculos entre el crecimiento económico y el desarrollo humano estaban fracasando para los habitantes de muchos países, cuyo desarrollo era desequilibrado, ya sean porque tenían buen crecimiento económico pero escaso desarrollo humano o porque tenían buen desarrollo humano pero un escaso o ningún crecimiento económico.

En términos generales este informe concluye que a medida que el mundo se adelante en el siglo XXl, en general se necesitará más crecimiento económico. Pero debe prestarse más atención a la estructura y a la calidad de este crecimiento para velar porque esté orientado al apoyo del desarrollo humano, la reducción de la pobreza, la protección del medio ambiente y la garantía de la sostenibilidad.

También en este documento se ha puesto claramente de manifiesto que no existe un vínculo automático entre crecimiento y desarrollo humano. Incluso cuando tal vinculo se establece, puede ir erosionándose gradualmente, a menos que se le refuerce en forma constante por medio de una gestión pública hábil e inteligente.

\section{CICLO VIRTUOSO, CICLO VICIOSO Y DESARROLLO ASIMÉTRICO EN LOS DEPARTAMENTOS DE COLOMBIA}

La debilidad en los eslabones de la cadena (crecimiento-desarrollo humano) característica de un buen número de departamentos de Colombia, tienen como efecto su inclusión en una categoría de desarrollo asimétrico con sesgo procrecimiento y con tendencia a un ciclo vicioso.

Se había comentado que una población mas sana y educada contribuye a mejorar la producción y el empleo económico; el resultado de lo anterior es la inclusión en una categoría de desarrollo asimétrico con sesgo prodesarrollo y con tendencia a un ciclo 
virtuoso, no obstante existen casos de regiones de Colombia donde no se corrobora lo anterior (un ejemplo es el departamento del Huila en el periodo 1994-2000).

Sin embargo como se mencionó, lo más probable es que estos casos de desarrollo asimétrico no persistan. Con el tiempo, la parte débil actuará como freno sobre la otra y se producirá un ciclo vicioso; o bien, si a lo largo del tiempo se fortalecen las vinculaciones, quizá mediante una modificación de las políticas, habrá un ciclo virtuoso.

En este epígrafe se seleccionaron 24 Departamentos de Colombia, para los que disponíamos de toda la información de IDH - PIB/H, para el periodo 1990-2005, posteriormente se calculó las variaciones respectivas entre años para estos dos indicadores, calculando las medias de variación para cada uno de los departamentos en este periodo, definiendo finalmente la media total para todos los departamentos del país en estos dos aspectos (ver anexo 4). El anterior ejercicio tiene como objetivo elaborar un plano cartesiano con la ubicación de la media en variación del IDH y $\mathrm{PIB} / \mathrm{H}$ de cada departamento, con respecto a la media total del país.

Esta ubicación clasifica el desarrollo de los departamentos en cuatro categorías?: la de ciclo virtuoso (un buen desarrollo humano refuerza el crecimiento, que a su vez promueve el desarrollo humano), la de ciclo vicioso (un comportamiento deficiente en materia de desarrollo humano tiende a traducirse en resultados mediocres en cuanto a crecimiento económico, lo que a su vez reduce los logros en desarrollo humano, y así sucesivamente) y dos categorías de desarrollo asimétrico, una con sesgo pro desarrollo humano (esto es, con fuerte desarrollo humano y escaso crecimiento económico) y otra con sesgo pro crecimiento económico (es decir, con escaso desarrollo humano y marcado crecimiento económico). El resultado del anterior ejercicio lo podemos ver en la grafica 1.

Como se puede observar en esta grafica no necesariamente un buen rendimiento en crecimiento económico esta relacionado con un buen rendimiento en desarrollo humano, tal es el caso de Santander y algunos otros departamentos. Por lo tanto esta hipótesis no queda corroborada gráficamente para ciertas regiones. De ahí el interés de los análisis de ámbito local y regional para detectar las causas de estas desviaciones respecto a la correlación de círculo virtuoso observada en otras regiones.

Para comprobar de mejor manera la evidencia empírica y teórica de Ranis, que concluye que ningún país que comienza en un sesgo pro crecimiento económico pudo pasar de allí a un ciclo virtuoso sino que todos ellos volvieron a caer en el círculo vicioso, sería mas conveniente disponer de datos para un periodo mayor al utilizado en

\footnotetext{
${ }^{7}$ Metodología propuesta por Ranis, Stewart y Ramírez en la relación crecimiento económico y desarrollo humano
} 
este trabajo, de esta manera lograríamos hacer comparaciones entre décadas, pero a nivel de departamentos en Colombia, solo existen datos a partir de 1990. Por lo tanto aparte del ya necesario análisis, esbozaremos la ubicación de cada departamento en este periodo con el objetivo de hacer recomendaciones. La ubicación con respecto a las categorías y ciclos en el periodo la podemos ver en la siguiente gráfica.

\section{Gráfico 1}

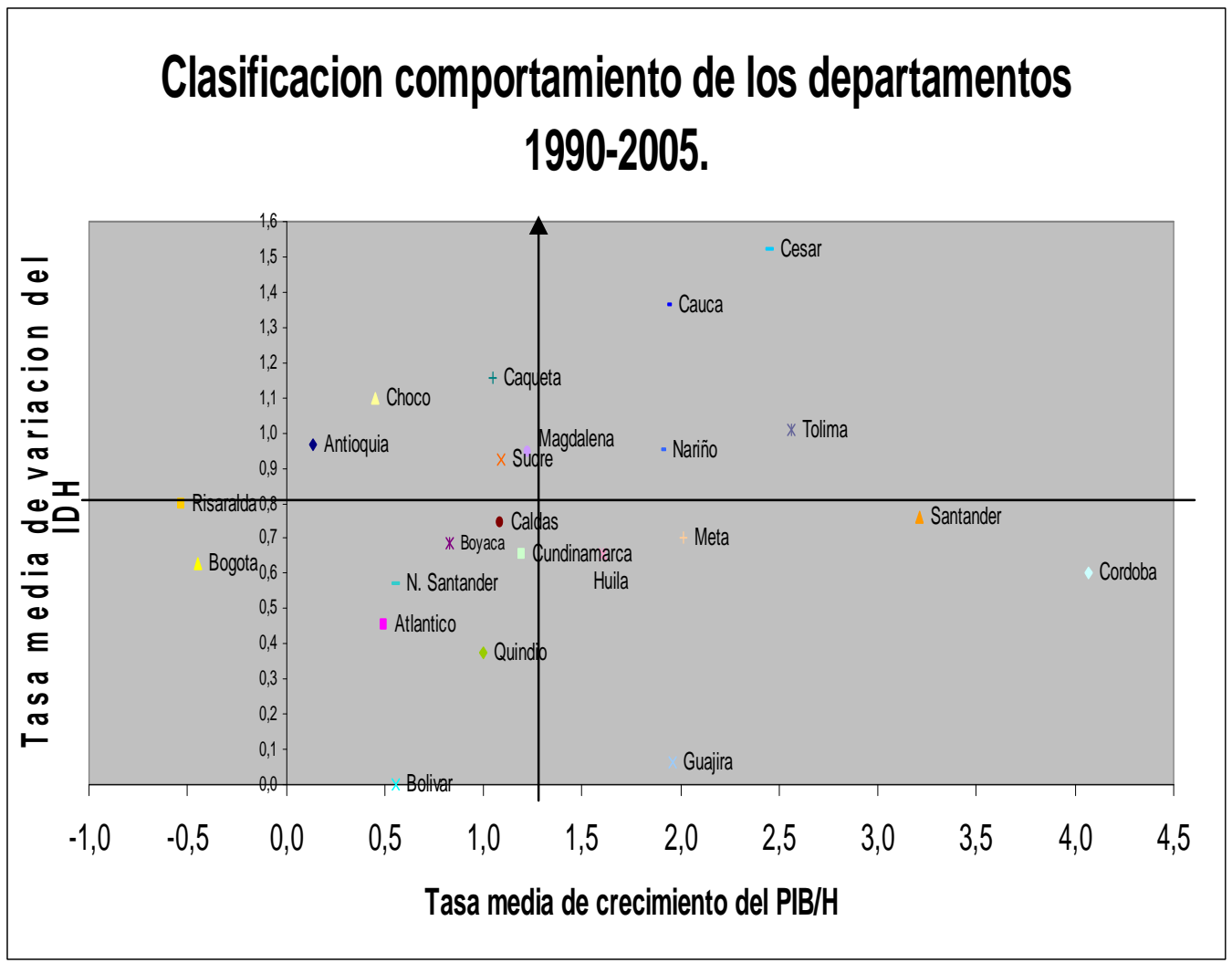

Fuente: Datos suministrados por las estadísticas anuales de planeación nacional

En conclusión podemos observar, que departamentos como Córdoba, Huila, Guajira, Meta y Santander, tienen la posibilidad de caer en un ciclo vicioso al estar ubicados en una categoría de desarrollo asimétrico con sesgo poscrecimiento, como lo han hecho nueve de los departamentos del país.

Por tal razón se recomienda a las administraciones de estos departamentos priorizar políticas de desarrollo humano, para en un próximo igual periodo, terminar en un ciclo virtuoso. 
Cuadro número 1: Ubicación de los departamentos de Colombia en el periodo 1990-2005 en ciclos y sesgos.

\begin{tabular}{|l|l|}
\hline \multicolumn{1}{|c|}{ DEPARTAMENTOS. } & \multicolumn{1}{c|}{$\mathbf{1 9 9 0 - 2 0 0 5}$} \\
\hline Antioquia & P. Desarrollo \\
\hline Atlántico & Vicioso \\
\hline Bolívar & Vicioso \\
\hline Boyacá & Vicioso \\
\hline Caldas & Vicioso \\
\hline Caquetá & Prodesarrollo \\
\hline Cauca & Virtuoso \\
\hline Cesar & Virtuoso \\
\hline Córdoba & Procrecimiento \\
\hline Cundinamarca & Vicioso \\
\hline Chocó & Prodesarrollo \\
\hline Huila & Procrecimiento \\
\hline La Guajira & P. Crecimiento. \\
\hline Magdalena & Prodesarrollo. \\
\hline Meta & P. Crecimiento. \\
\hline Nariño & Virtuoso. \\
\hline Norte Santander & Vicioso. \\
\hline Quindío & Vicioso \\
\hline Risaralda & Vicioso \\
\hline Santa Fe de Bogota D. C. & Vicioso \\
\hline Santander & P. Crecimiento. \\
\hline Sucre & Prodesarrollo \\
\hline Tolima & Virtuoso \\
\hline Valle & P. Desarrollo \\
\hline & \\
\hline
\end{tabular}

Fuente: Datos suministrados por las estadísticas anuales de planeación nacional. 


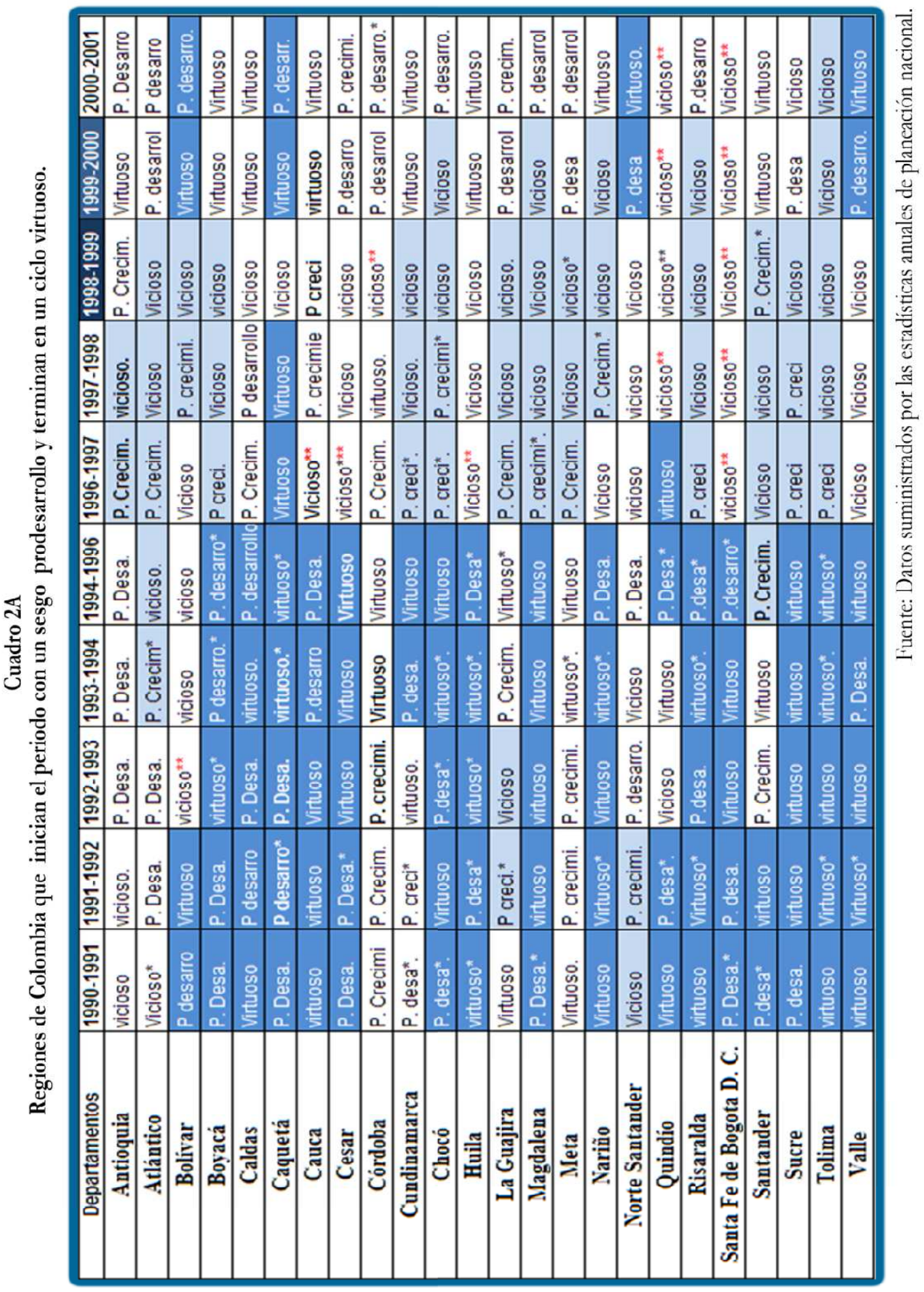




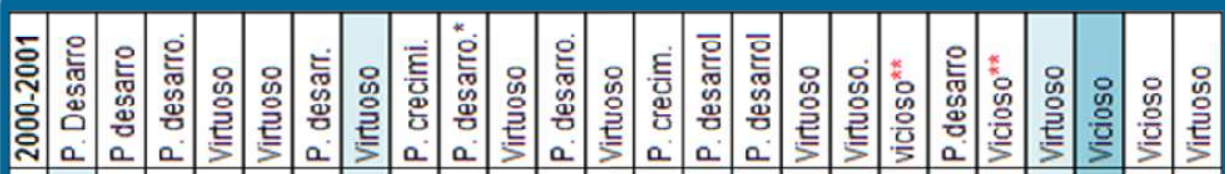

흐

\%

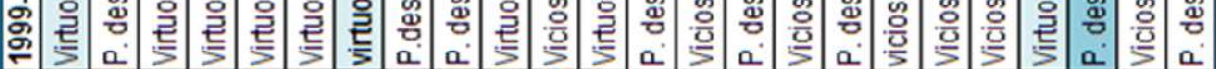

ริ 틍

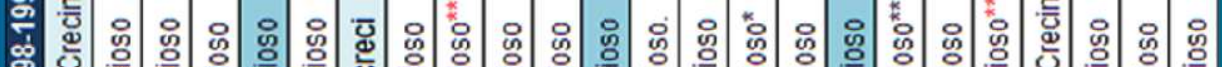

\%

F

F

寻

备 \%

을

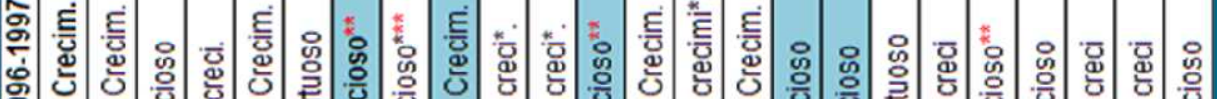

\%

웅

ڤ

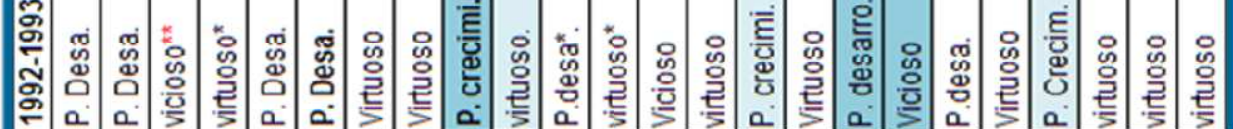

.ำ.

.ב

$\stackrel{\vartheta}{\rightleftarrows}$ 旁

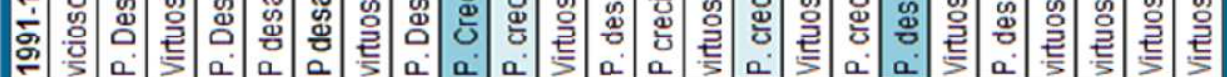

हี

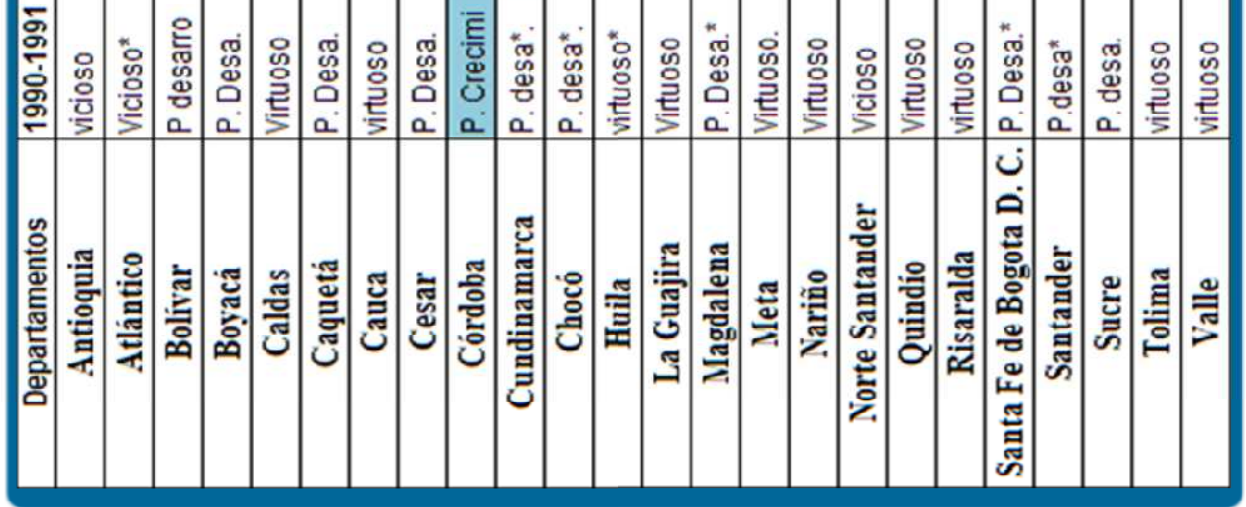


Regiones y periodos donde se corrobora la hipotesis.

Regiones y periodos donde no se corrobora la hipotesis.

Periodo de crisis economica $\left({ }^{* *}\right)$.

Periodo despues de una crisis economica

Periodo de buen desempeño (aunque por debajo de la media) *

Fuente: Datos suministrados por las estadísticas anuales de planeación nacional.

Al hacer el análisis año a año, estar en un sesgo procrecimiento para algunos departamentos no implica terminar en un ciclo vicioso, o por el contrario estar en un sesgo prodesarrollo no implica terminar en un ciclo virtuoso, incluso en algunos pocos casos se pasa con continuidad de una categoría a otra a través de los años, es posible que esta ultima situación se de porque obedece a que se trata de un número menor de regiones, de un periodo de análisis muy corto, o porque la semejanza entre ellos es mayor, lo que significa que incluso variaciones leves pueden conducir a cambios de categoría.

Para la elaboración del cuadro 2 A y B, he colocado un asterisco (*) a aquellas situaciones o periodos en las que se logra un buen desempeño en desarrollo humano y crecimiento económico, aunque su porcentaje de variación no este por encima de la media nacional en el periodo de registro.

Un ejemplo de lo anterior es cuando existe un incremento medio nacional del $4 \%$ en (IDH Y PIB/H), pero al compararlo con una región que cuenta con un aumento del $3,88 \%$ en ambos aspectos, no necesariamente este departamento registra un mal desempeño y por tanto un ciclo vicioso, existe en el, un ciclo virtuoso de buen desempeño aunque no supere la media, o por el contrario si la variación media nacional es de $-4 \%$ en estos aspectos, tener un registro de $-2 \%$ en desarrollo humano y crecimiento económico, no significa que exista un ciclo virtuoso en esta región. En los anteriores cuadros podemos apreciar estas situaciones.

Como podemos ver en el cuadro $2 \mathrm{~A}$, la gran mayoría de las regiones durante el periodo de análisis cumplen las hipótesis planteadas en este trabajo. Entre todos los casos presentados resalto el de Caquetá, que tras obtener un periodo de sesgo prodesarrollo (1990-1993), termina en un ciclo virtuoso en el año 1993 y seguidamente hasta 1998. Otra de las situaciones es la de Antioquia, que tras estar en un sesgo 
procrecimiento en el periodo 1996-1997, termina en un ciclo vicioso en el periodo 1997-1998.

En un pequeño número de regiones (ver cuadro 2B) no se corrobora las hipótesis planteadas, es el caso de Meta, que tras estar en un sesgo procrecimiento en el periodo 1991-1993, termina en un ciclo virtuoso (esto quiere decir que en esta región y periodo existió un mayor gasto social y una mejor distribución de la riqueza). En el otro extremo se encuentra una región como la de Cauca, que en el periodo 1993-1996 se encontraba en situación de sesgo prodesarrollo y termina en un ciclo vicioso, es posible que esto se de al no tener una buena articulación entre su recurso humano capacitado y saludable con el sistema productivo.

Una crisis económica puede explicar los cambios bruscos entre categorías, un ejemplo de ello es la presentada en Colombia en el año 1999, donde la gran mayoría de regiones, sin importar la categoría o ciclo virtuoso en el que se encontraban antes de la misma, terminan en un ciclo vicioso durante la crisis. Sin embargo la gran mayoría de regiones después de esta gran crisis pasan a un ciclo virtuoso o sesgo prodesarrollo, demostrando de alguna manera la buena capacidad de recuperación, pero este repunte es insuficiente para rescatar el gran terreno perdido. Los departamentos que no se han recuperado de la misma forma son Chocó, Magdalena, Nariño, Quindío, Risaralda y Tolima.

\section{CONCLUSIÓN}

Puede que una evaluación año a año no sea el más conveniente para este tipo de análisis, no obstante se puede ver que en gran parte de las regiones y durante el periodo de estudio, sí se cumple con las afirmaciones planteadas al inicio de este epígrafe. Pero al presentarse situaciones en las que no ocurre (así sean mínimas), no podemos afirmar que necesariamente todas las conclusiones a las que han llegado han llegado Ranis Stewart y Ramírez tengan validez universal, siendo imprescindible seguir profundizando en este tipo de investigaciones.

\section{REFERENCIAS}

- $\quad$ Ainsworth, M. Beegle, K. (1995): The impact of female schooling on fertility and contraceptive use: A Study of Fourteen sub-Sabaran Countries LSMS Working Paper, No 10 110, Washington, D.C., Banco Mundial.

- Alesina, A. y Rodrik, D. (1994): Distributive politics and economic growth, Quarterly Journal of Economic, vol.109, N 2, Cambridge, Massachusetts, MIT Press. 
- Alesina, A. y R. Perotti (1994): The political economy of growth: A critical survey of the recent literature, The World Bank Economic Review, vol. 8, N3, Washington, D. C; Banco Mundial.

- Behrman, J.R. (1993): The economic rationale for investing in nutrition in developing countries, World Development, vol. 21, N 11, Oxford, Reino Unido, Pergamon press.

- Behrman, J.R. (1996): The impact of health and nutrition on education, The World Bank Research Observer, vol. 11, N 1, Washington, D.C., Banco Mundial.

- Birdsall, N., D. Ross y R. Sabot (1995): Inequality and growth reconsidered: Lessons from East Asia, The World Bank Economic Review, vol. 9, N 3, Washington, D.C, Banco Mundial.

- Edwards, S. (1993): Openness, trade liberalization, and growth in developing countries, Journal of Economic Literature, vol. 31, $\mathrm{N}^{\circ}$ 3, Nashville, Tennessee, American Economic Association.

- Krueger, A. (1978): Foreign Trade Regimes and Economic Development: Liberalization Attempts and Consequences, Cambridge, Massachusetts, Ballinger.

- La Fuente, M. y Sanz P. (2001): "Participación de los pobres en los frutos del crecimiento", Revista CEPAL número 76.

- Michaely, M. (1977): Exports and growth: An empirical investigation, Developing Economics, vol. 4, N 1, Ámsterdam, Países Bajos, North-Holland.

- PNUD. (1996): Informe sobre desarrollo bumano, página 1.

- Psacharopoulos y otros, G. (1992): Poverty and Income Distribution in Latin America: The Store of the 1980, Washington, D.C., Banco Mundial.

- Ram, R. (1985): Exports and economic growth: Some additional evidence, Economic Development and Cultural Change, vol. 33, $\mathrm{N}^{\circ}$ 2, Chicago, Illinois, The University of Chicago Press.

- Rana, P. (1988): Exports, policy changes and economic growth in developing countries after the 1973 oil shock, Developing Economics, vol. 28, N³ 3, Amsterdam, Países Bajos, North- Holland.

- Ranis, G. Stewart, F. y Ramirez, A. (2000): "Economic growth and human development", Word Development, vol. 28, No 2, Oxford, Reino Unido, Pergamon Press.

- Ranis, G. Stewart, F. y Ramírez, A. (2002): "Crecimiento y desarrollo humano en Latinoamérica", Revista CEPAL, número 78.

- Rosenzwei, M.R. (1995): ¿Why are there returns to schooling?, The American Economic Review, Vol. 85, No 2, Nashville, Tennessee, American Economic Association

- Schultz, T. W. (1975): The value of the ability to deal with disequilibria, Journal of Economic Literature, vol. 13, $\mathrm{N}^{\circ}$ 3, Nashville, Tennessee, American Economic Association.

- Welch, F. (1970): Education in production, Journal of political economy, vol.78, No 1, Chicago, Illinois, The University of Chicago Press. 